\title{
Pyogenic liver abscess in children: Diagnosis and treatment at the teaching hospital Gabriel Touré, Bamako, Mali
}

\author{
Coulibaly Yacaria, Amadou Issa*, Keita Mamby, Diaby Souleymane Gaoussou, Konaté Madiassa, \\ Diallo Gangaly \\ Department of Pediatric Surgeon, University Hospital of Gabriel Touré, Bamako, Mali \\ Email: isamt77@yahoo.fr
}

Received 28 January 2013; revised 27 February 2013; accepted 9 March 2013

Copyright (c) 2013 Coulibaly Yacaria et al. This is an open access article distributed under the Creative Commons Attribution License, which permits unrestricted use, distribution, and reproduction in any medium, provided the original work is properly cited.

\begin{abstract}
Purpose: To determine diagnostic and therapeutic problems of pyogenic liver abscess in our hospital. Method and material: We conducted a retrospective study from January 2006 to December 2010 of all children aged from 0 - 15 years admitted and treated for pyogenic liver abscesses. Amoebic liver abscesses and other hepatobiliary disorders were not considered. Results: Pyogenic abscesses accounted for $\mathbf{5 0}$ cases. The mean age of patients at presentation was $2.4 \pm 0.78$ years (range from 6 months to 15 years). Sex ratio was 2.8 for boys. Abdominal pain was the primary reason for consultation in 27 cases $(54 \%)$. Fever has been noted in 42 patients $(84 \%)$ and the patient general state was altered in 13 patients $(26 \%)$. Escherishia coli was the most frequent bacteria found at pus. The pus was sterile in 25 cases $(50 \%)$. The ultrasonographic percutaneous drainage was performed in 33 patients $(66 \%)$, open surgery in $6(12 \%)$ and antibiotics alone in 11 others (22\%). The short outcomes were simple in 36 patients $(\mathbf{7 2} \%)$ while pain and fever persisted in 12 others (24\%). Two patients (4\%) died from sepsis soon after the operating room. Improving the frequency of early diagnosis will depend on education of clinicians about the need for clinical suspicion aided by ultrasound.
\end{abstract}

Keywords: Pyogenic Liver Abscess; Children; Diagnosis; Treatment; Mali

\section{INTRODUCTION}

Liver abscess remains a formidable diagnostic and therapeutic problem in developing countries. Pyogenic liver abscesses (PLA) account for almost $80 \%$ of all liver

*Corresponding author. abscesses in the developed world and are most often polymicrobial [1].

Untreated, it is uniformly fatal due to ensuing complications such as sepsis, or peritonitis secondary to rupture of the abscess cavity into the pleural or peritoneal cavities [2].

The clinical presentations in many cases of PLA are non-specific in nature and are difficult to diagnose early [3]. Effective management involves elimination of both abscess and the underlying source. In these last years in Mali, the incidence of hepatic abscesses is rising but the diagnosis is late. We have conducted this study to determine diagnostic and therapeutic problems of this affection in our hospital.

\section{MATERIEL AND METHOD}

We conducted a retrospective study from January 2006 to December 2010 of all children aged from 0 - 15 years admitted and treated for pyogenic liver abscesses. Amoebic liver abscesses and other hepatobiliary disorders were not considered. The diagnosis of pyogenic abscess was made on the appearance and the non-chocolate color of pus. Blood count, blood glucose, blood culture, the pyoculture of sampling, HIV status, Widal-Felix laboratory tests and abdominal ultrasound were requested in all patients. Chest radiography was performed in clinical respiratory signs. Data were collected from patients' medical records including consultation reports, hospitalizations' entries and operative summaries. These data were then entered and analyzed on the software Epi Info 6.4.fr and $\mathrm{Chi}^{2}$ test used for comparison with $\mathrm{p}<0.05$.

\section{RESULTS}

From January 2006 to December 2010, 5785 admissions were made to the pediatric surgery department including 109 cases of liver abscess. Pyogenic abscesses accounted 
for 50 cases. The mean age of patients at presentation was $2.4 \pm 0.78$ years (range from 6 months to 15 years). Sex ratio was 2.8 for boys. Abdominal pain was the primary reason for consultation in 27 cases (54\%) and in 16 cases (32\%) patients were referred by the pediatric medical after abdominal ultrasound. The average time for consultation was $16 \pm 1.8 \mathrm{j}$ with a range of 6 to 30 days.

No medical and surgical history had been found in 42 cases (84\%). A notion of gastroenteritis was found in 7 patients and sepsis in a single case. The majority of patients were treated for fever at a medical center before being sent to the hospital. Fever has been noted in 42 patients (84\%) and the patient general state was altered in 13 patients (26\%). The functional and physical signs are listed in Table 1. A blood count to neutrophil leukocytosis was found in 37 patients (74\%) and anemia in 36 (72\%). HIV status was negative in all cases. The Widal-Felix serology was positive in 2 cases (4\%). Blood cultures had been positive in 3 cases (6\%). Blood glucose was normal in all patients. Examination of pus has found Escherishia coli in 9 cases, Staphylococcus aureus in 4 cases, Klebsiella sp in 5 cases, Haemophilus influenzae in 4 cases, Enterobacter cloacae in 3 cases. The pus was sterile in 25 cases (50\%).

After examination, ultrasound has found ruptured abscess in 2 cases and four patients were operated for peritonitis due to rupture of liver abscesses. The abscess was in collecting process in 8 cases and has already collected in 36 cases. No biliary, portal or tumor cause has been found. Abscess was located in the right lobe in 22 cases (44\%), left lobe in 19 cases (38\%) and multifocal in 9 cases (18\%). It was smaller than $60 \mathrm{~mm}$ in 11 cases (25\%), between 60 and $80 \mathrm{~mm}$ in 25 cases (57\%) and greater than $80 \mathrm{~mm}$ in 8 cases (18\%). Chest radiograph was performed in 32 patients and showed an elevated hemidiaphragm in 6 patients and pleural effusion in 3 cases.

Table 1. Sign.

\begin{tabular}{ccc}
\hline Signs & $\mathrm{N}$ & $\%$ \\
\hline Upper quadrant tenderness & 43 & 86 \\
Abdominal pain & 34 & 68 \\
Nausea/vomiting & 31 & 62 \\
Hepatomehaly & 25 & 50 \\
Crackles & 7 & 14 \\
Cough & 7 & 14 \\
Abdominal contracture & 6 & 12 \\
Diarrhoea & 6 & 12 \\
Constipation & 4 & 8 \\
\hline
\end{tabular}

The ultrasonographic percutaneous drainage was performed in 33 patients (66\%), open surgery in 6 (12\%) and antibiotics alone in 11 others (22\%). At percutaneous drainage, the average amount of aspirated pus was $158 \pm$ $119 \mathrm{ml}$ (range of 50 to $474 \mathrm{ml}$ ) with an average of $4 \pm 1.8$ sessions. Medical treatment for all patients was started on a dose of $80 \mathrm{mg} / \mathrm{kg}$ of cetriaxone and $30 \mathrm{mg} / \mathrm{kg}$ of metronidazole up to apyrexia, and then treatment continued orally up to 15 days. The average hospital stay was $13 \pm 4.9$ days (range from 6 to 23 days). The short outcomes were simple in 36 patients (72\%) while pain and fever persisted in 12 others (24\%). Two patients (4\%) died from sepsis soon after the operating room. A wound infection was noted in 2 patients. Comparisons between type of treatment and complications had shown Chi2 = $5.585 \mathrm{p}=0.95$, between abscesses' diameters and complication found Chi2 $=8.793 \mathrm{p}=0.99$ and between abscesses sites and complications found Chi2 $=6.308 \mathrm{p}=$ 0.975 . At ultrasound a month later, a residual cavity was detected in 12 cases. At 6 months, except for 8 lost patients, all others had no lesion on ultrasound.

\section{DISCUSSION AND COMMENTS}

When performing this study we were faced with a number of problems. It was impossible to do blood and pyocultures in our hospital. Punctures were made in our structure and samples sent to others with the risk of lengthening the period of biological fluid keeping.

PLA remains a major diagnostic and therapeutic challenge, despite advances in diagnostic technology and new strategies for treatment. A combination of non-specific systemic symptoms is the most common presentation; including fever and rigors, nausea and vomiting, right upper quadrant pain, anorexia, weight loss, weakness and malaise. Less frequently reported symptoms include cough or hiccups due to diaphragmatic irritation, with referred pain to the right shoulder. Patients with small solitary lesions usually have a more insidious course with associated weight loss, fatigue and anaemia of chronic disease. In our study upper quadrant pain was the most frequent sign. Fever has been the second sign. In tropical context fever can be seen in many diseases. All of our patients arrived after a long circuit and many antimalarial drug and antibiotics. That will be contributing to lengthen time of consultation. In our series average time of consultation was 16 days. This long time was also confirmed in adult patients by Senegalese authors [4]. This delay in diagnosis increases frequency of complications which can be local or general. In our series abscesses rupture has been seen in 6 patients and two died for sepsis. Ultrasound was reported as $85.8 \%$ sensitivity of liver abscess at emergency department [5]. In this study it was done in all our patients because of its coast and was diagnostic in all of them. In resource-lim- 
ited places it can help in confirming the diagnosis in the presence of a triad of Fontan.

The abscess was most common in right lobe (44\%). Previous studies also reported similar findings [6,7]. Preferential laminar drainage of the superior mesenteric vein to right lobe may explain this frequency. The laboratory features are also non-specific for diagnosis of PLA. Widal-Felix laboratory test to diagnose typhus salmonellas was positive only in two cases and confirmed by hemoculture. Recently despite the high rate of salmonellosis and HIV in our country, no ground research has been positive.

Organisms recovered from liver abscesses vary greatly but generally reflect bile or enteric flora. Reasons for the variability include differences in antibiotics before culture, cultures techniques or patients population. The most frequent organism isolated from abscess aspirate was Escherichia coli. Recent "Western" series suggested that Escherichia coli is still the most common causative organism [8,9]. Many of the large case series are from South East Asian institutions, where a large proportion of pyogenic liver abscesses are caused by Klebsiella pneumoniae [10]. The low positive rate of bacterial culture (50\%) was probably related to the use of empirical antibiotics treatment prior to the drainage procedure. This rate of $50 \%$ was not statistically different from the 55\% of Zhu et al. [6] in China ( $\mathrm{p}>0.05)$.

Prognostic factors include the patients' age, multiplicity of abscesses and organisms and the presence of associated malignant or immunosuppressive disease. Survival from pyogenic abscess has improved in recent years with earlier diagnosis and treatment. All patients were empirically commenced on broad spectrum intravenous antibiotics at presentation. The most commonly used empiric antimicrobial regimen was ceftriaxone and metronidazole. This was changed according to the sensitivities of organisms cultured from each patient's abscess aspirate or blood. In resource-limited countries percutaneous drainage is a good alternative in the treatment of abscesses and fluid collections in the abdomen. Percutaneous drainage has been used in $66 \%$ of our patients with unifocal and single abscess of more than $6 \mathrm{~cm}$. In our 4 median ranges it allowed abscess emptying without any major complications. This was confirmed by Wong et al. [11] with 80 patients who underwent percutaneous aspiration.

Operative management of liver abscesses may be associated with significant morbidity and mortality [12,13]. In our study, the mortality was $4 \%$. This was not statistically different to those of authors in the literature $[13,14]$. Unlike mortality, morbidity is more difficult to compare between studies, because of differences in completeness of data collection in retrospective studies and therefore tends to be under-reported. Complications such as biliary fistula, leakage, and haemorrhage following percutaneous drainage or aspiration are known, but uncommon, complications.

Our complications were not related to abscesses' site and diameter and to the type of treatment ( $p>0.05)$. The mean hospital stay was 13 days.

\section{CONCLUSION}

The prognosis of patients with PLA can be further improved by early diagnosis and effective treatments at the early stage of PLA progression. Improving the frequency of early diagnosis will depend on education of clinicians about the need for clinical suspicion aided by ultrasound.

\section{REFERENCES}

[1] Krige, J.E. and Beckingham, I.J. (2001) ABC of diseases of liver, pancreas, and biliary system. British Medical Journal, 322, 537-540. doi:10.1136/bmj.322.7285.537

[2] Heneghan, H.M., Healy, N.A., Martin, S.T., Ryan, R.S., Nolan, N., Traynor, O. and Waldron, R. (2011) Modern management of pyogenic hepatic abscess: A case series and review of the literature. BMC Research Notes, 4, 80. doi:10.1186/1756-0500-4-80

[3] Johannsen, E.C., Sifri, C.D. and Madoff, L.C. (2000) Pyogenic liver abscesses. Infectious Disease Clinics of North America, 14, 547-563. doi:10.1016/S0891-5520(05)70120-3

[4] Dieng, M., Diop, B., Cisse, M., Konate, I., Ka, O., Dia, A. and Toure, C.T. (2009) Drainage des abcès du foie par la technique de l'hépatotomie à minima. Revue Médecine Tropicale, 69, 475-476.

[5] Lin, A.C., Yeh, D.Y., Hsu, Y.H., et al. (2009) Diagnosis of pyogenic liver abscess by abdominal ultrasonography in the emergency department. Emergency Medicine Journal, 26, 273-275. doi:10.1136/emj.2007.049254

[6] Zhu, X.J., Wang, S.H., Jacob, R., Fan, Z., Zhang, F. and Ji, G.Z. (2011) 10-year retrospective analysis of clinical profiles, laboratory characteristics and management of pyogenic liver abscesses in a Chinese hospital. Gut Liver, 5, 221-227. doi:10.5009/gnl.2011.5.2.221

[7] Foo, N.P., Chen, K.T., Lin, H.J. and Guo, H.R. (2010) Characteristics of pyogenic liver abscess patients with and without diabetes mellitus. The American Journal of Gastroenterology, 105, 328-335. doi:10.1038/ajg.2009.586

[8] O’Farrell, N., Collins, C.G. and McEntee, G.P. (2010) Pyogenic liver abscesses: Diminished role for operative treatment. Surgeon, 8, 192-196. doi:10.1016/j.surge.2010.01.001

[9] Lee, K.T., Wong, S.R. and Sheen, P.C. (2001) Pyogenic liver abscess: An audit of 10 years' experience and analysis of risk factors. Digestive Surgery, 18, 459-465. doi:10.1159/000050194

[10] Pang, T.C.Y., Fung, T., Samra, J., Hugh, T.J. and Smith, R.C. (2011) Pyogenic liver abscess: An audit of 10 years' 
experience. World Journal of Gastroenterology, 17, 1622-1630 doi:10.3748/wjg.v17.i12.1622

[11] Wong, W.M., Wong, B.C., Hui, C.K., Ng, M., Lai, K.C., Tso, W.K., Lam, S.K. and Lai, C.L. Pyogenic liver abscess: Retrospective analysis of 80 cases over a 10-year period. Journal of Gastroenterology and Hepatology, 17, 1001-1007. doi:10.1046/j.1440-1746.2002.02787.x

[12] Christein, J.D., Kendrick, M.L. and Que, F.G. (2006) What affects mortality after the operative management of hepatic abscess? Health Promotion Board (Oxford), 8, 175-178. doi:10.1080/13651820500477738
[13] Tan, Y.M., Chung, A.Y., Chow, P.K., Cheow, P.C., Wong, W.K., Ooi, L.L. and Soo, K.C. (2005) An appraisal of surgical and percutaneous drainage for pyogenic liver abscesses larger than $5 \mathrm{~cm}$. Annals of Surgery, 241, 485-490. doi:10.1097/01.sla.0000154265.14006.47

[14] Bosanko, N.C., Chauhan, A., Brookes, M., Moss, M. and Wilson, P.G. (2011) Presentations of pyogenic liver abscess in one UK centre over 15-years period. The Journal -Royal College of Physicians of Edinburgh, 4, 13. doi:10.4997/JRCPE.2011.104 